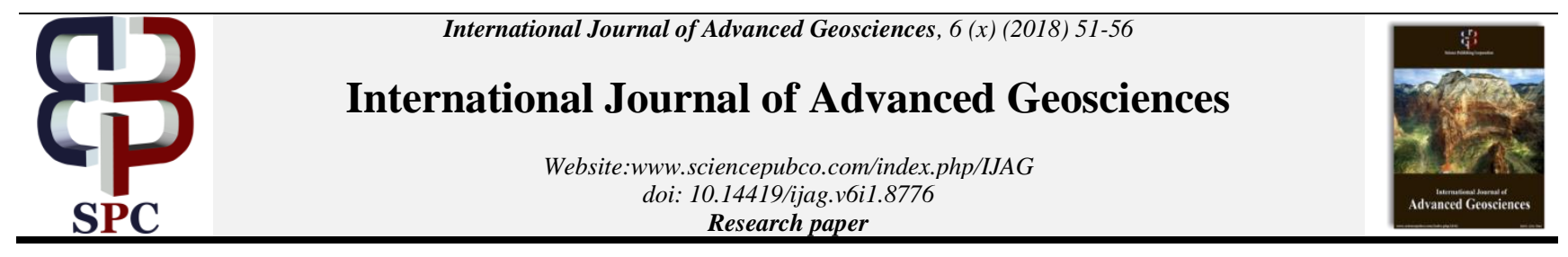

\title{
Spectral analysis of Rayleigh waves in south-eastern parts of Niger delta, Nigeria
}

\author{
Aniwetalu Emmanuel ${ }^{1 *}$, Ilechukwu Juliet ${ }^{2}$, Oguadinma Vivian ${ }^{2}$, Chiadikobi Kingsley ${ }^{3}$, Nnaji Ezechimelu ${ }^{1}$ \\ ${ }^{1}$ Department of Geology and Mining Godfrey Okoye University, Enugu, Nigeria \\ ${ }^{2}$ Department of Geological Sciences, Nnamdi Azikwe University Awka, Nigeria \\ ${ }^{3}$ Chukwuemeka Odumegwu Ojukwu University, Uli Anambra State, Nigeria \\ *Corresponding author E-mail: emmymega4@gmail.com
}

\begin{abstract}
Interference of ground roll energy on true seismic reflection records has continued to pose a serious challenge to exploration geophysicists. In view of this, amplitude and power spectra of the Rayleigh waves which are the precursor of the ground roll energy were derived from over 70 raw monitor records and plotted as a function of frequency. The objective is to determine the locus of ground energy in the seismic records, analyse their dispersion pattern and suggests viable ways of suppressing them. The results of the amplitude spectrum plots revealed that Rayleigh waved exhibit oscillatory behavior with very high-amplitude values, which correspond to the locus of ground roll energy. This energy is confined to very low frequency range of about 4-9Hz. The Power spectrum which was given as the square of the amplitude as a function of frequency showed appreciable lobes of breaths of the ground roll energy of about $0.5-0.7 \mathrm{~cm}$ and their trend of dispersions. The power spectrum plots revealed several peaks excluding the early peaks that are direct indication of ground roll energy. The plots showed pronounced and constant decline in energy levels with increasing frequency and reaching very low decibel values of $-60 \mathrm{Db}$ to $-80 \mathrm{Db}$ at frequency range of $50 \mathrm{~Hz}$. This indicates that the environment is dispersive in nature which probably results from velocity layering. This is a precursor to seismic noise which among others can be suppressed in the field by designing filters with sharper cut off characteristics.
\end{abstract}

Keywords: Amplitude Spectrum; Dispersion Pattern; Ground Roll; Power Spectrum and Rayleigh Waves.

\section{Introduction}

Ground roll is any event that is added to the seismic signals in the course of generating, recording and processing of the seismic data. The vertical component of the ground roll is composed of Rayleigh's waves. Over the years, ground roll has continued to pose a very serious challenge to the hydrocarbon exploration because of its interference with true seismic reflection events that may give useful information of the subsurface geology of an area. The reliability on this seismic data for identification and interpretation of the subsurface geologic features are strongly dependent upon the quality of the seismic records. The presence of ground rolls in any seismic records affects the quality of the data.

Removal or suppression of the ground roll energy has become one of the greatest challenges of exploration geophysicists today. In view of this, many studies have been undertaken on the possible ways to suppress the ground roll during data acquisition. Most scholars maintained that ground roll elimination from seismic traces is possible by exploiting their specific characteristics. Aniwetalu and Anakwuba (2015) used quantitative techniques to derive the character of the ground roll from raw seismic data and also defined appropriate attenuation strategy for their suppression during data acquisition. Ebeniro (1993) studied the dispersive behaviour of Rayleigh's waves. He stated that Rayleigh's waves could be dispersive, especially when different frequency components are travelling at different velocities. He concluded that all seismic waves exhibit dispersive behaviour in a medium which displays attenuation. Several authors have suggested that Ray- leigh's waves have their most natural classifications with respect to their dispersion trends (Mooney and Kaasa, 2005; Sengbush, 1983; Aniwetalu and Anakwuba 2015).In their studies; they determined the dispersive or non dispersive Rayleigh waves by analyzing the relationship between phase velocity, group velocity and frequency. The properties of Rayleigh wave in Delaware basin in west Texas, and Southern New Mexico was concisely discussed by Dobrin (1951). He observed that wave speed and amplitude of ground roll can be related to the velocities of the layer near the surface.

In this study, our focus is to derive the amplitude spectrum, power spectrum, frequency of Rayleigh's waves and use their relationship to determine the locus of ground roll energy and its dispersion trend in the study area. The term spectrum here describes the variation of certain qualities such as energy or amplitude as a function of some parameters normally frequency or wavelength. The fundamental aspect of this qualitative analysis of ground roll character is the determination of time or frequency domain that provides the huge understanding of signal properties. Using Fourier's series, seismic trace can be described in the conventional time domain or transformed into the frequency domain. Fourier spectrum has a very distinct uniqueness in wave signal analysis. A signal frequency domain expression is its spectrum. A periodic signal can be defined either in time domain (as a function) or in the frequency domain (as a spectrum). The mathematical expression is useful and of interest, but a graph of frequency- (domainrepresentation amplitude versus frequency) is most useful for the interpretation of the physical phenomenon (Oppenheirmar and Lyer 1980, Sheriff and Geldart 1982). The amplitude is the size of change in the 
oscillating variable, with each oscillation within an oscillating system. The analysis of wave amplitude, power spectrum and frequency components can be used to enhance the knowledge of ground roll energy and dispersion trend in an environment.

\subsection{Location and geologic setting the study area}

The study area is geographically located in the South-Eastern region of the Niger Delta and confined between latitude of $3^{\circ}$ $3^{\prime}$ and $3^{\circ} 7^{\prime}$ 'south and longitude $5^{\circ} 3^{\prime}$ 'and $3^{\circ} 4^{\prime}$ 'east (Fig.1). The area is situated in Central Onshore depobelt of Niger Delta and characterised with beaches, coastal flats, rivers, swamps and lagoon. The area is subject to seasonal flooding with high water level during the wet season and poor drainage system during the dry season.
Some parts of the study area fall on the Mangrove swamp while some lie within the floodplain of geomorphic unit of Niger Delta. The geomorphic unit of Niger delta is very extensive and complex (Fig.2). Therefore, to understand the character of the ground roll in this environment, it is imperative to know the accumulation pattern of the sediments and their geomorphic relations. Short and Stauble (1967) described how sediment of Niger Delta were transported by Niger and Benue Rivers and latter redistributed by longshore currents. The weathered layer of these sediments has a disproportionately large effect on the travel times of seismic wave which is the precursor of ground roll effect on seismic records (Fig.3).

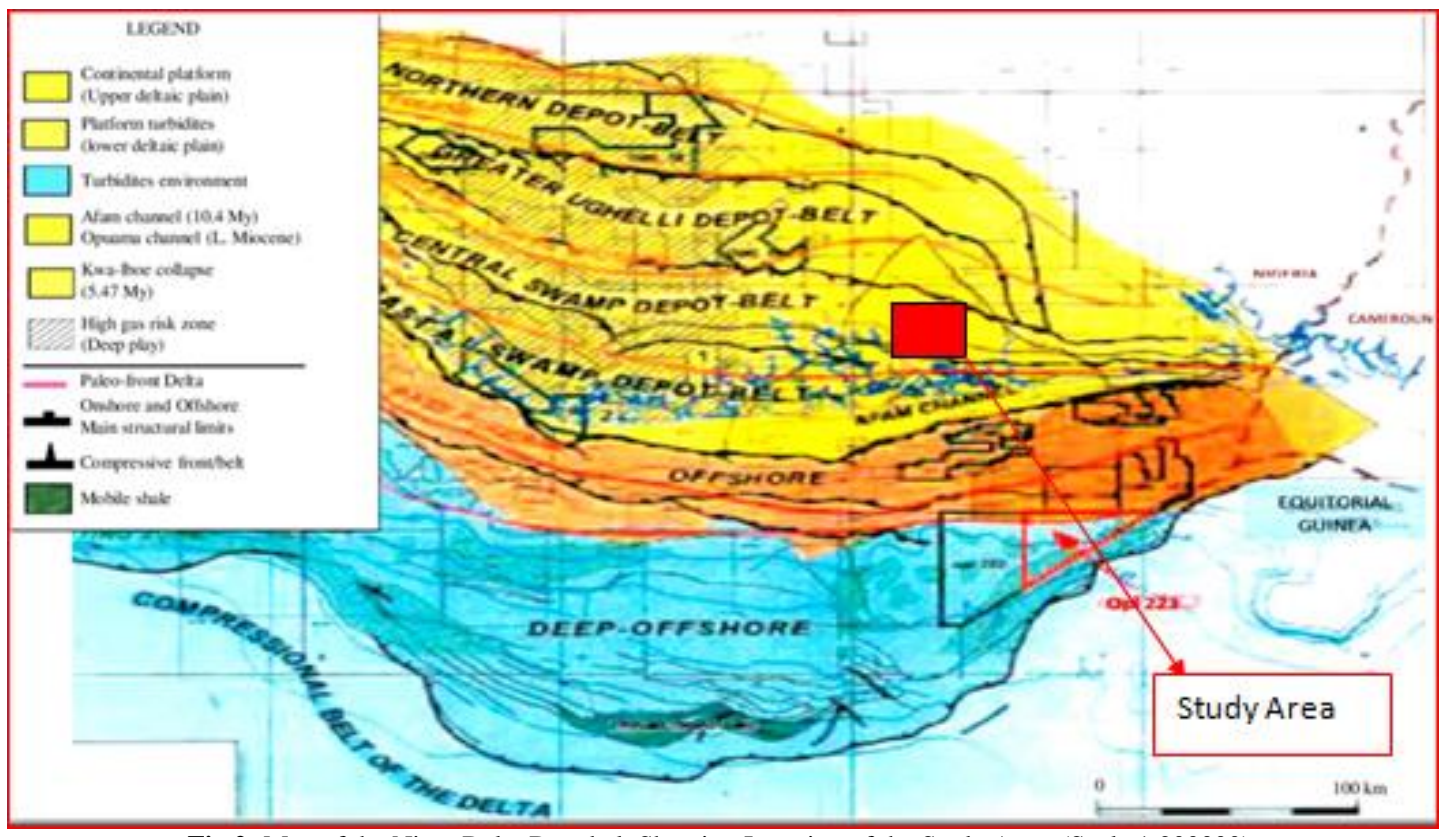

Fig.2: Map of the Niger Delta Depobelt Showing Location of the Study Area. (Scale 1:200000).

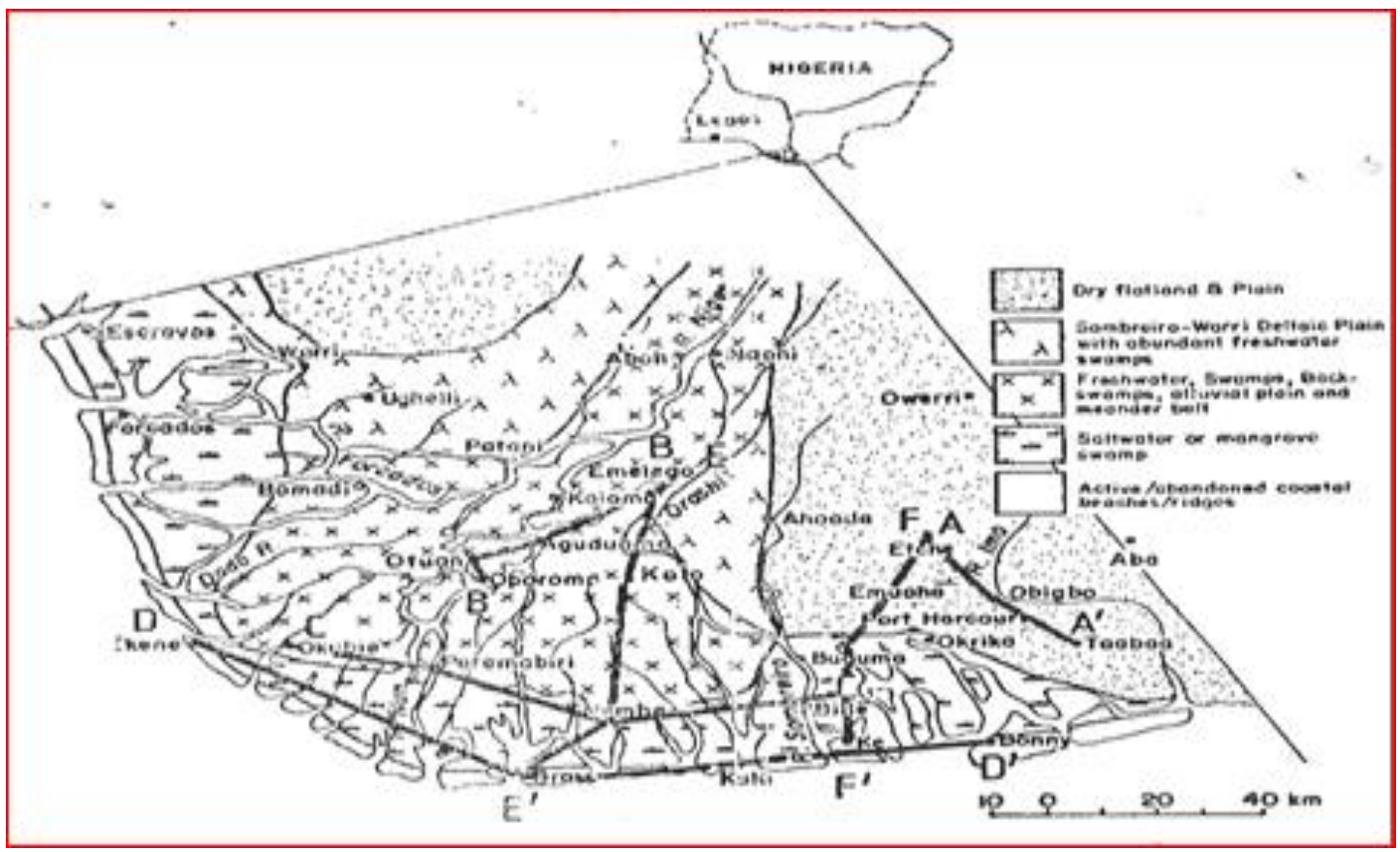

Fig. 2: The Major Geomorphologic Units of the Niger Delta Basin (Modified by Akpokodje, 1987). 


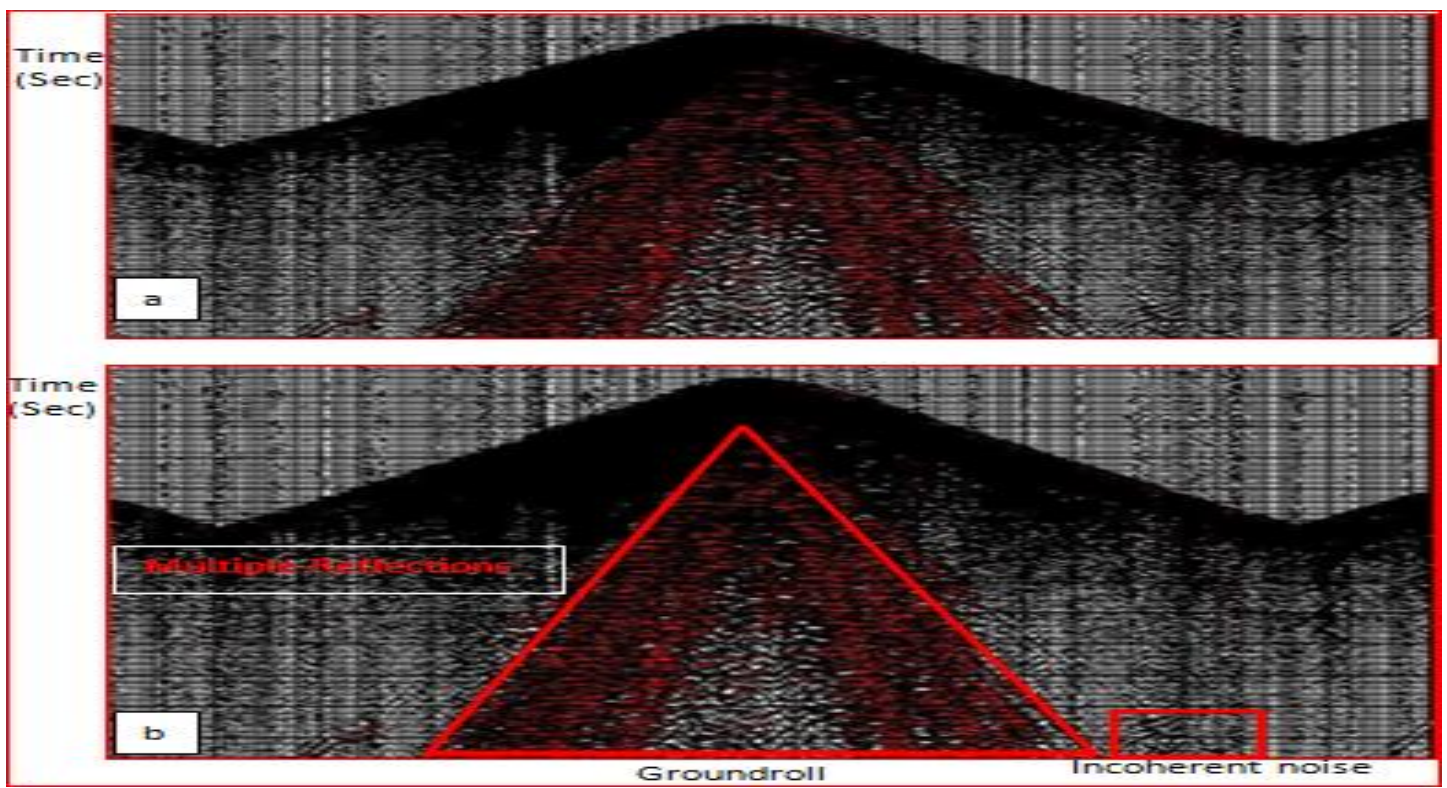

Fig. 3: A) A Typical Example of Noisy Shot Profile (B) the Marked Noise Trains

\section{Materials and methods}

Material used for this study was acquired by the united geophysical crew during routine seismic data acquisition. The considerable parts of the study area were covered with water. This necessitates the use of both geophones and hydrophones. Swath shooting technique which consists of some mutually perpendicular lines (receiver and short lines) layout on the ground was adopted. This was defined in line with the structural trend of the Niger Delta. The receiver lines consisted of 120 groups of source detectors. Eighteen geophones/hydrophones phones were planted per group. Interval of $50 \mathrm{~m}$ and $2.28 \mathrm{~m}$ were used per group and adjacent geophones respectively. Seismic records were generated through seismic shooting using dynamite as an energy source. Seismic reflection record obtained during this exercise was remarkably dented with ground roll (Fig.4). However, our focus is to derive the magnitude of change in amplitude and power spectra of these ground roll and establish their dispersion trend. The magnitude of change in the oscillating variable is referred to as amplitude. The amplitude spectrum can be derived by expressing the amplitude as a function of frequency. This was carried out using Matlab program, which computed and compared the amplitude of signals as a function of frequency. The total power spectrum is given as the square of amplitude as a function of frequency. Such spectrum does not contain any phase information (Parasnis 1979).This implies that once the amplitude spectrum of a given event is known, its power spectrum could be deduced. More also, the same set of parameters that control the output of amplitude spectral analysis would also hold for power spectral. The following sets of parameters were supplied to the system for analysis. First record, last record, trace interval, first trace, last trace, start time, end time, sample rate, and scale. The number of records analyzed were chosen after due consideration to the data points that were representative of the study area. However, traces in each record were chosen, bearing in mind the onset of ground roll on the records. For instance, in Fig.4, the onset and concentration of the ground roll energy were from 4-9 seconds and between traces of 350-418. This made it possible to obtain as close as possible the magnitude and strength or intensity of ground roll energy relative to the other events on the record. The system output percentage amplitude on the vertical axis while corresponding frequencies are displayed on the horizontal axis. The power spectral was expressed in a decibel. The choice of Decibel scale rests on the fact that the unit is normally used to express the power ratio. Moreover, the scale offers a convenient method in the measurement of the ratio. The system output the spectrum with the power displayed in the vertical axis, and the corresponding frequency displayed on the horizontal axis.

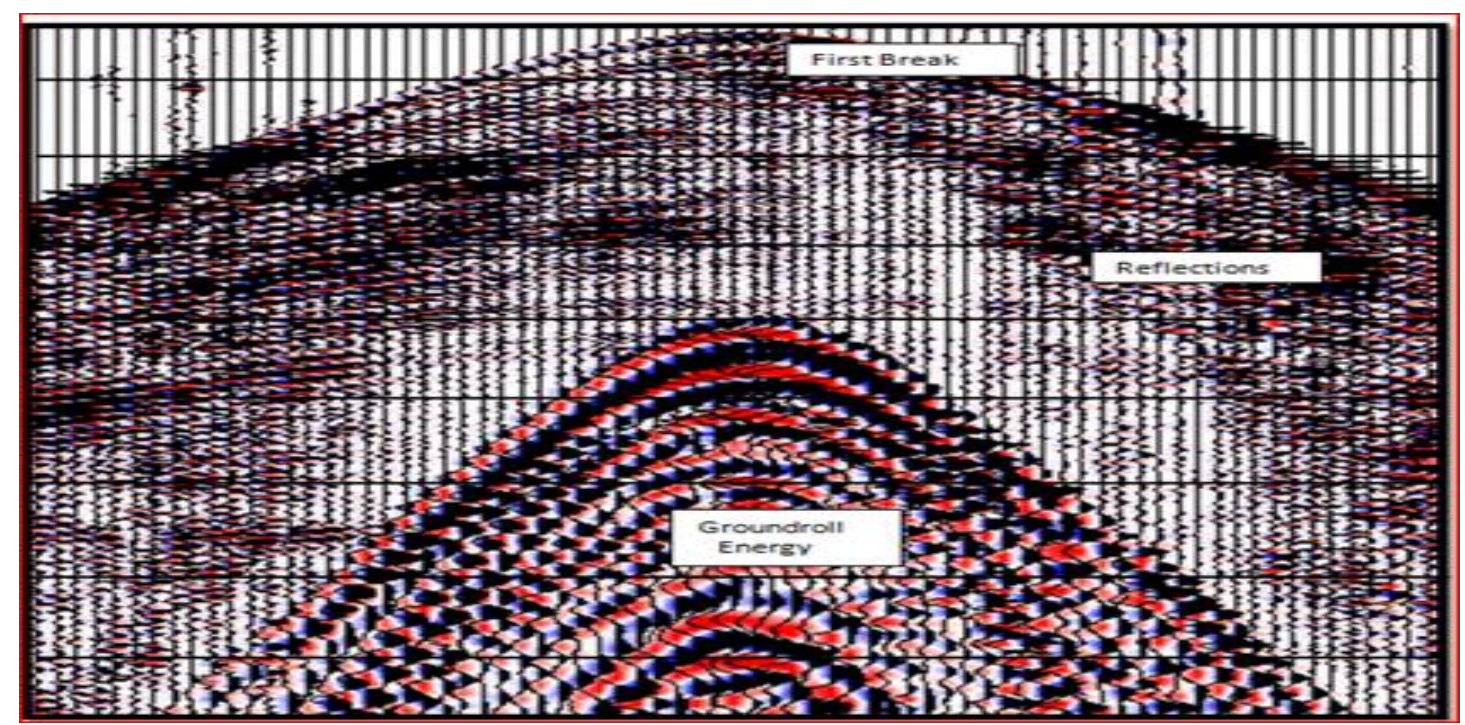

Fig. 4: Ground Rolls on Seismic Data (after Aniwetalu and Anakwuba 2015). 


\section{Results and discussion}

The amplitude spectrums of some monitor records of the study area are shown in Fig.3 and Fig.4. In the plots, the amplitudes of the events were expressed as a function of frequency. In general, the plots exhibit an oscillatory character, reaching the maximum amplitude level at frequency between $4.0-9 \mathrm{~Hz}$. The progressive shift of the more significant part of the amplitude spectrum towards the lower frequency and corresponding broadening of the wavelength is attributed to the rapid or faster attenuation of the higher-frequency components. The high-amplitude regions observed in the plot corresponded to the locus of ground roll energy, and they are confined to very low frequency range. A critical inspection of these amplitude displays indicated that the Rayleigh waves are confined to a narrow frequency band $(4-9 \mathrm{~Hz})$ around the spectral peaks. Applegate (1982) showed that frequency range of ground roll could be estimated for geological environment by spectrum of the short windows dominated by noise. Therefore, the amplitude spectral analyses of the monitor records revealed that Rayleigh's wave energy is accompanied with high amplitude and low frequency values. The Power spectrum which is defined as a spectrum giving the square of the amplitude as a function of frequency also showed ground roll energy and their dispersion trend. Figs 5 and Fig.6 showed power spectra plots of time monitor records of the study area. The total power was expressed in decibel (DB) and also plotted as a function of frequency. Characteristically, the plots exhibit an oscillatory character, showing pronounced and constant decline in energy levels with increasing frequency and reaching very low decibel value of $-80 \mathrm{db}$ at frequency of $50 \mathrm{~Hz}$ (Fig.5) and -60db at frequency of $50 \mathrm{~Hz}$ (Fig.6). Therefore, power spectrum when plotted on decibel scale, is identical to its amplitude spectrum. Increasing Decibel values indicate an increasing amplitude and power. The shift of more significant parts of the power spectrum towards the lower frequencies is the reflection of the amplitude distribution within the record. The power spectrum plot of the monitor records has several peaks on the spectrum excluding the early peaks that are direct indication of ground roll energy. These peaks indicate that good ground coupling was obtained at these frequencies, and that resonance frequency of the column corresponds to these frequencies. The early peaks in the power spectra represent the ground roll energy.

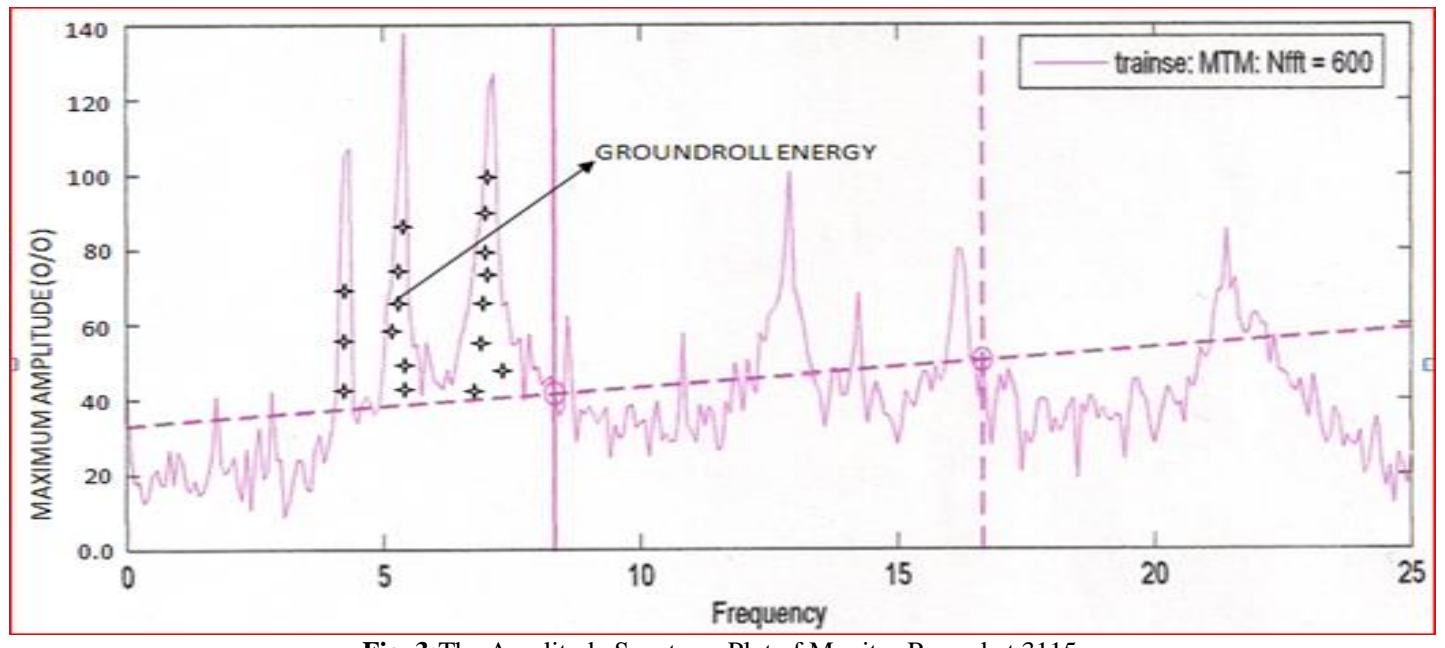

Fig. 3:The Amplitude Spectrum Plot of Monitor Record at 3115.

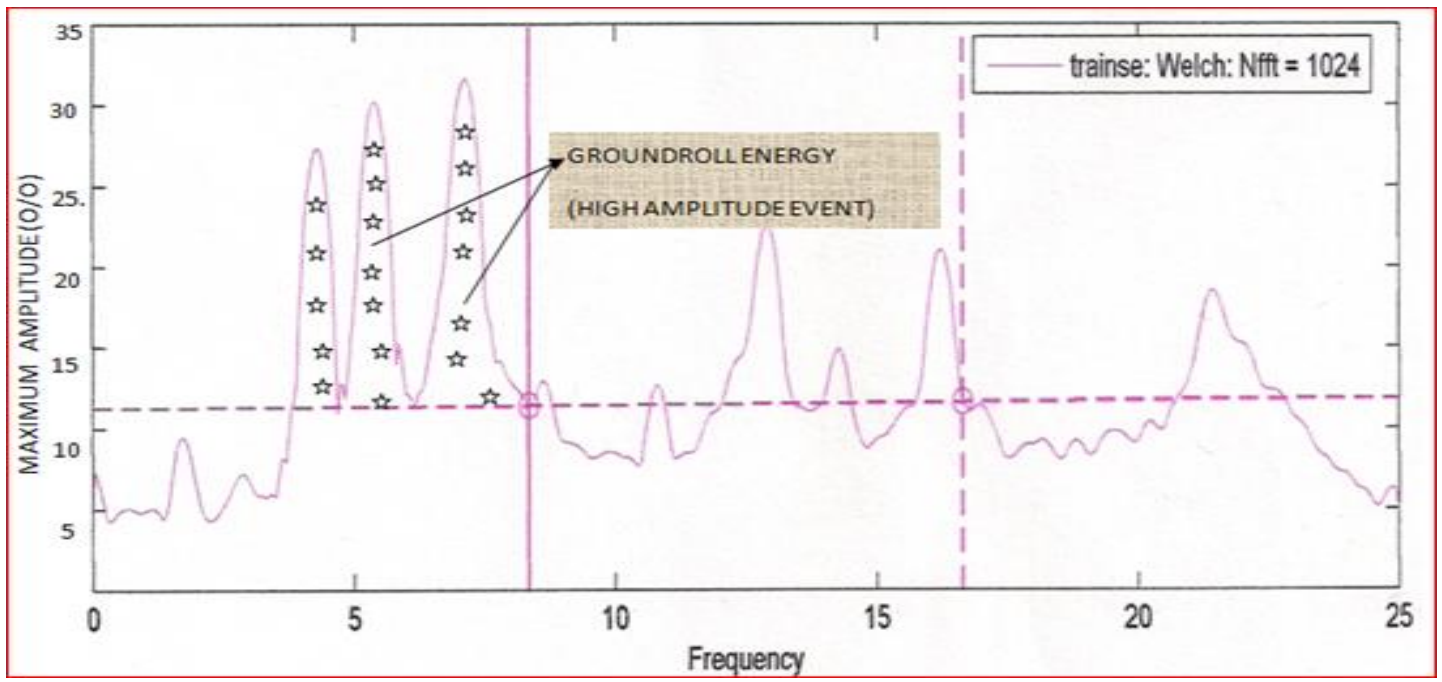

Fig. 4: Amplitude Spectrum Plot of Record 3120 of the Study Area.

Like amplitude spectrum, power spectra are confined between frequency range of $4-9 \mathrm{~Hz}$ with lobes of breadth of $0.7 \mathrm{~cm}$ at $-30 \mathrm{Db}$ (Fig.5) and $0.5 \mathrm{~cm}$ at $-40 \mathrm{db}$ (Fig.6). Although most explosive's sources in seismic exploration emit minimum phase signals or wavelets. An implicit assumption usually made in seismic reflection interpretation is that the source generates a single shortduration pulse. On this assumption, the input and output wavelet approach a spike (i.e. wavelet having a flat amplitude spectrum with unit amplitude density). In this approach, non-dispersive events (waves with the equal amount of all frequencies in phase in which group velocity is equal to phase velocity) will manifest as a spike and dispersive events will emerge as a lobe of appreciable breadths as evident in Fig. $5(0.7 \mathrm{~cm})$ and Fig.6 $(0.5 \mathrm{~cm})$.). Therefore, it was clear that the power spectral plots of this study area are far from the spike model. Therefore, events are observed as dispersive in nature. This suggests that ground roll energy in the 
study area is dispersive in nature. Dispersion of waves occurred when seismic pulse propagates through layered earth sections or where there is inhomogeneities that scatter seismic waves. Anstey (1993) suggested that all seismic waves were dispersive in a medium who exhibited an elastic attenuation. Most scholars agreed that the cause of the Rayleigh wave dispersion is probably the presence of velocity layering. This is because in the multilayered medium, Rayleigh waves propagate in discrete modes. The phase and group velocity of each mode depends on frequency and at most frequencies, these velocities are significantly different. How- ever, in comparing the dispersive Rayleigh waves with non dispersive Rayleigh's waves, the major difference worthy to note is that, on any given offset trace. The dispersive Rayleigh wave exhibited a long duration. Different frequency components of the waves are propagating in different velocities but in the case of non dispersive Rayleigh's wave, phase and group velocities are identical and independent on frequency. The dispersive nature of the Rayleigh wave is dependent on weathered layer of the earth,

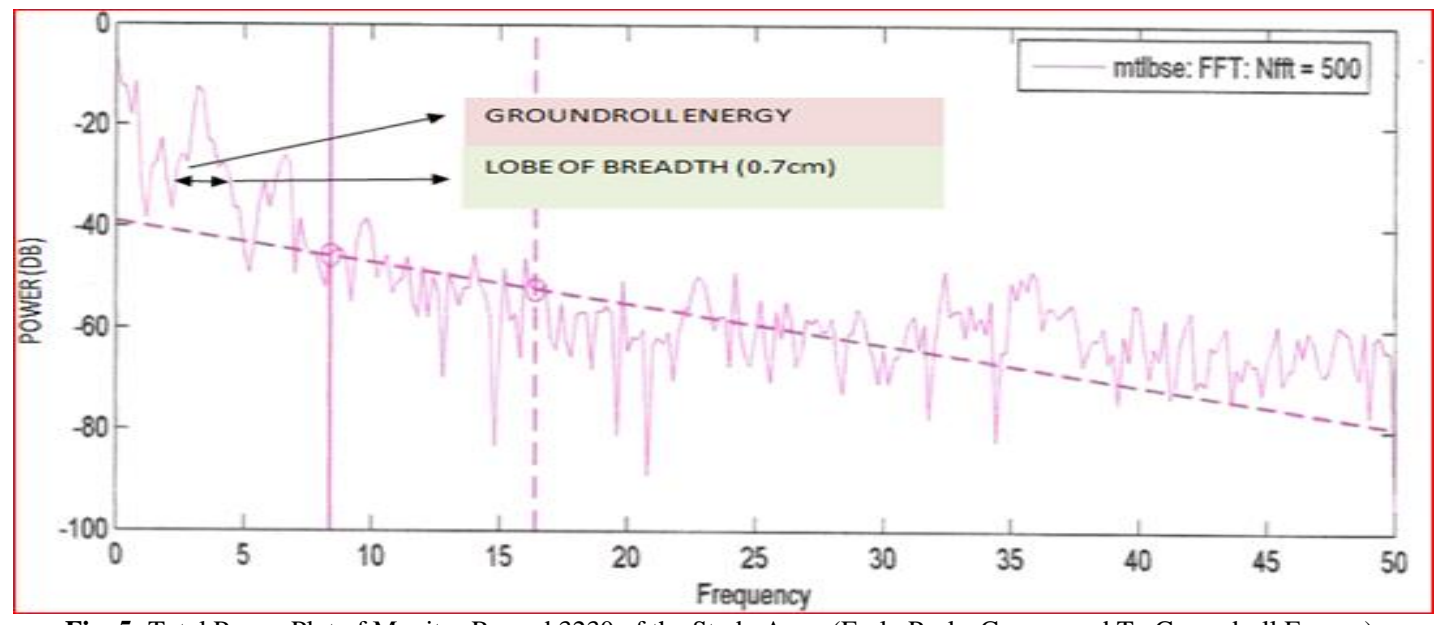

Fig. 5: Total Power Plot of Monitor Record 3230 of the Study Area. (Early Peaks Correspond To Groundroll Energy).

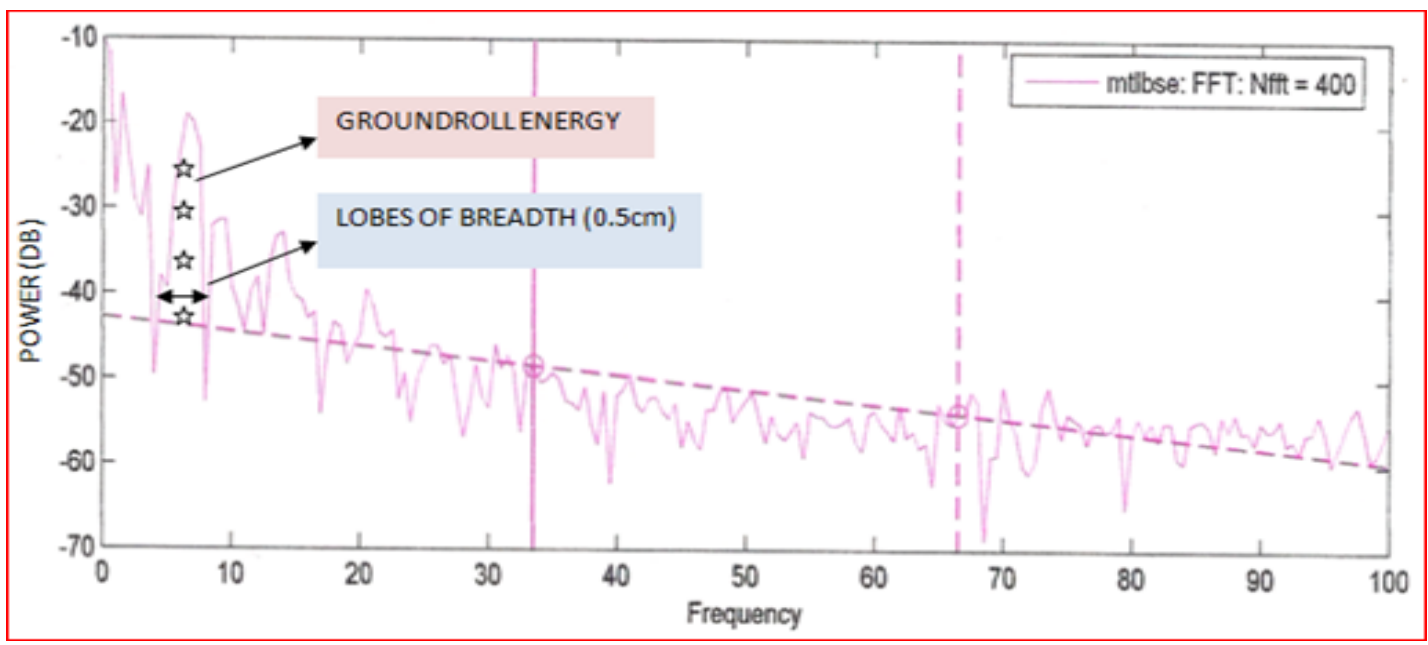

Fig. 6: Total Power Plot of Monitor Record 3032 of the Study Area.

Generally referred to as geologically recent unconsolidated sediments that also have very low velocity values. This layer is very high in absorption of seismic energy due to much structural friction being encountered by wave signals as they wiggle through the layers. The thickness of these layers varies from about 5 to $45 \mathrm{~m}$ or more while velocity may vary from 250 to $1000 \mathrm{~m} / \mathrm{s}$ (Aniwetalu and Anakwuba 2015).Uko (1991) observed that the variation of thickness and velocity of the weathered layer within the layer could both be horizontal and vertical and more also, rapid and erratic. The changes in velocity and thickness in this layer have the huge effect of the travel times of the seismic pulse. It acts as a variable low-pass filter or a high-cut filter on the amplitude spectrum which is capable of distorting wavelet shape. Weathered layers have very high impedance contrast, and waves can be trapped in this layer thus causing multiple reflections that could result to mode conversion. However, the variations in the physical properties of the low-velocity layers are the major cause of wave dispersion and deterioration of land seismic data.

\section{Conclusion}

In this study, amplitude and power spectrum of some monitor records of the study area were derived and expressed as a function of frequency. The aim was to derive the locus of ground roll energy and determine their dispersion pattern in the study area. The plot of amplitude and power spectrum revealed that the locus of the ground roll energy is confined within the low-frequency range of $4-9 \mathrm{~Hz}$ with high amplitude and power spectrum values. The Power spectrum which was derived from square of the amplitude as a function of frequency, exhibit an oscillatory character, with appreciable lobes of breadth of about $0.5-0.7 \mathrm{~cm}$ which is an indication of dispersive environment. It also showed the constant decline in energy levels with increasing frequency and reaching very low decibel value of $-80 \mathrm{db}$ at frequency of $50 \mathrm{~Hz}$. The dispersive nature of this environment probably results from velocity layering. Because in multilayered medium, waves of different frequency component's propagates at different velocities thus causing wave dispersion and deterioration of seismic records in large scale. 
Based on the above observations, following conclusions were drawn

- Since the ground roll energy concentrates within a frequency band that is much lower than the energy of the signal, the design of filters with sharper cut off a characteristic is necessary.

- Adopting smaller charges or burying the source below weathered layers could also aid in suppressing the ground roll in seismic records.

- Array with omnidirectional attenuation characteristic should be used in the study area in view of scattered Rayleigh's waves.

- Similar studies should be carried out in other geomorphic units of the Niger Delta to enable a more meaningful or regional correlation of event characteristics.

\section{Acknowledgements}

We wish to express our profound gratitude to the Department of Shell liaison Office University of Port Harcourt, Rivers State for providing the data and for their technical assistance during data analysis.

\section{References}

[1] Aniwetalu E.U. and Anakwuba E.K (2015). Quantitative characterization of groundroll (Rayleigh waves in the Western Niger Delta, Nigeria, Arch. Appl. Sci. Res. 7 (3) 21-36.

[2] Akpokodje E.A (1987). The engineering geological characteristics and classification of the superficial soils in the Niger Delta, Eng. Geol. 23, 198-211https://doi.org/10.1016/0013-7952(87)90090-1.

[3] Anstey N.A. (1993). Whatever happened to ground roll? Geophys. Leading Edge Exploration, 3:4-15.

[4] Applegate J.K. (1982). High resolution seismic study in the Gas Hills Uranium district, Wyoming. Geophysics 47, 1355 1361.https://doi.org/10.1190/1.1441286.

[5] Dobrin M.B. (1986). Introduction to Geophysical Prospecting. 3rd Edn, McGraw Hill Inc., NewYork

[6] Ebeniro, J.O. (1993). Important of multiple suppression in seismic exploration. Journal of mining and geology 29, 183-193.

[7] Mooney H.M and R.A. Kaasa, (2005). Air waves in engineering seismology. Geophysics, 54:290-301.

[8] Oppenheirma D.V and Lyer H.M. (1980). Frequency wave number analysis of geothermal micro seismic at Norris Geyer Basin, Yellow National park Wyoming. Geophysics 45, 952 963.https://doi.org/10.1190/1.1441099.

[9] Parasins D.S (1966). Principles of Applied Geophysics. 2nd Edn. Ghapman and Hall, London.

[10] Sengbush R.L. (1983). Seismic exploration Method IHRDC., Boston.https://doi.org/10.1007/978-94-011-6397-2.

[11] Sheriff R.E and Geldart L.P (1982). Exploration Seismology 1, Cambridge University Press.

[12] Uko E.M (1991). Weathering structure of eastern Niger delta. NAPE Bull.6, 67-74. 